\title{
Editorial on "Biochemical and Epigenetic Insights into L-2-Hydroxyglutarate, a Potential Therapeutic Target in Renal Cancer"
}

\author{
Spencer Hart, Gopal Gupta \\ Department of Urology, Loyola University Medical Center, Maywood, IL, USA \\ Correspondence to: Spencer T. Hart, MD. Loyola University Medical Center, 2160 S. First Ave. Bldg 54, Rm 247, Maywood, IL 60153, USA. \\ Email: spencer.hart@lumc.edu. \\ Provenance: This is an invited Editorial commissioned by Section Editor Xiao Li (Department of Urology, Jiangsu Cancer Hospital \& Jiangsu \\ Institute of Cancer Research \& Nanjing Medical University Affiliated Cancer Hospital, Nanjing, China). \\ Comment on: Shelar S, Shim EH, Brinkley GJ, et al. Biochemical and Epigenetic Insights into L-2-Hydroxyglutarate, a Potential Therapeutic Target \\ in Renal Cancer. Clin Cancer Res 2018;24:6433-46.
}

Submitted Oct 31, 2018. Accepted for publication Nov 20, 2018.

doi: $10.21037 / \mathrm{atm} .2018 .11 .43$

View this article at: http://dx.doi.org/10.21037/atm.2018.11.43

Renal cell carcinoma remains a common entity with approximately 65,340 new cases diagnosed in 2018. Given the insidious onset of disease, approximately $25-30 \%$ of patients will present with metastatic disease and require systemic therapy (1). Fortunately, the last two decades has seen the development of a number of targeted therapies including mammalian target of rapamycin (mTOR) inhibitors as well as tyrosine kinase inhibitors (TKIs) which are commonly used as first and second line therapies. Targeted therapy has offered a new alternative to toxic cytokine therapy, but overall response rates remain low. Analysis of the COMPARAZ trial comparing sunitinib versus pazopanib showed similar overall response rates of $25 \%$ for sunitinib and $31 \%$ for pazopanib (2). While there has been great interest in checkpoint blockade as a new avenue for therapy with the results of the recently published CheckMate 214 trial, the objective response rate remains low at 29\% (3). Given that only a fraction of patients with metastatic disease will respond to first line and subsequent therapies, the need for new drug targets for the treatment of advanced renal cancers is clear.

In this issue of Clinical Cancer Research, Shelar et al. describe a thorough in vitro and in vivo analysis of L-2-hydroxyglutarate (L-2-HG), its role in tumorigenesis, and its potential as a new therapeutic target in the treatment of renal cancers (4). To establish the role of L2HG in tumorigenesis, a L-2-HG dehydrogenase (L2HGDH) knockdown model was produced using immortalized renal epithelial cells with relatively high L2HGDH expression. This produced an in vitro migratory phenotype akin to that of renal cell cancers when high levels of L-2-HG were present. Similarly, treatment of renal epithelial cells with excessive exogenous L-2-HG produced a similar phenotype, highlighting the pivotal role of L-2HG as the mitigating factor. In an effort to highlight the importance of L-2-HG's in the tumor phenotype, transfection of wild type L2HGDH into L2HGDH deficient tumor cells demonstrated a reduced migratory phenotype. Restoration of function in an in vivo mouse model also demonstrated reduced growth rates on tumor explantation. Taken together with the Kaplan-Meier curve for patients with low L2HGDH, and consequently, higher L-2-HG levels, this argues for L-2$\mathrm{HG}$ as an appealing target for patients with renal cancers in improving overall survival.

The authors also demonstrated the potential role of carbon sources for L-2-HG in the in vitro setting as well as the role of malate dehydrogenase in increasing L-2-HG accumulation. These seem fewer appealing targets given the potential ramifications of systemic therapy given the ubiquitous nature of these enzymes in glucose metabolism. While the in vitro data presented is appealing, it begs the question of the consequences of targeting these in the in vivo model. This may also be the case for L-2-HG, but these targets in general have yet to be explored from a therapeutic standpoint. 
Despite these concerns, we believe the author's findings present an important new avenue in the understanding and treatment of renal cancers that warrants further exploration. While it is unlikely that drug therapies targeted at L-2$\mathrm{DH}$ would supplant existing therapies, it may provide an important adjunct in the appropriately selected patient.

\section{Acknowledgements}

None.

\section{Footnote}

Conflicts of Interest: The authors have no conflicts of interest to declare.

Cite this article as: Hart S, Gupta G. Editorial on "Biochemical and Epigenetic Insights into L-2-Hydroxyglutarate, a Potential Therapeutic Target in Renal Cancer". Ann Transl Med 2018;6(Suppl 2):S105. doi: 10.21037/atm.2018.11.43

\section{References}

1. Siegel RL, Miller KD, Jemal A. Cancer statistics, 2018. CA Cancer J Clin 2018;68:7-30.

2. Motzer RJ, Hutson TE, Cella D, et al. Pazopanib versus sunititnib in metastatic renal-cell carcinoma. NEJM 2013;369:722-31.

3. Motzer RJ, Tannir NM, McDermott DF, et al. Nivolumab plus ipilimumab versus sunitinib in advanced renal-cell carcinoma. NEJM 2018;378:1277-90.

4. Shelar S, Shim EH, Brinkley GJ, et al. Biochemical and Epigenetic Insights into L-2-Hydroxyglutarate, a Potential Therapeutic Target in Renal Cancer. Clin Cancer Res 2018;24:6433-46. 